\title{
A THEORY FOR THE SQUARE GUARDED HOT PLATE-A SOLUTION OF THE HEAT CONDUCTION EQUATION FOR A TWO LAYER SYSTEM*
}

\author{
$\mathrm{By}$ \\ I. G. DONALDSON \\ Dominion Physical Laboratory, Lower Hutt, New Zealand
}

Summary. A theoretical treatment is given for the action of the guarded hot plate apparatus used for measuring conductivities. The flow of heat through the metal hot plate itself, and the effects of temperature unbalance between hot plate and guard plate are both taken into account. The temperature at any point in the sample or in the heater plate may be determined. The theory is comprehensive and any of the dimensions or controlling parameters may be varied in the calculation.

Introduction. Although the thermal conductivity of insulating materials has been determined for many years with the guarded hot plate apparatus, it was not until recently that any attempts have been made to assess theoretically the errors associated with the use of this apparatus. Without the benefit of theoretical assessments the standards that have been specified by the ASTM [1], RILEM [2] and the British Standards Institution [3] have from necessity been drawn up relatively arbitrarily. Thus they differ to some extent one from another and in the light of recent theoretical and experimental work, do not appear to be sufficiently stringent for the $1 \%$ accuracy often demanded of the guarded hot plate apparatus.

Figure 1 illustrates the layout of a typical "guarded hot plate". Essentially, two
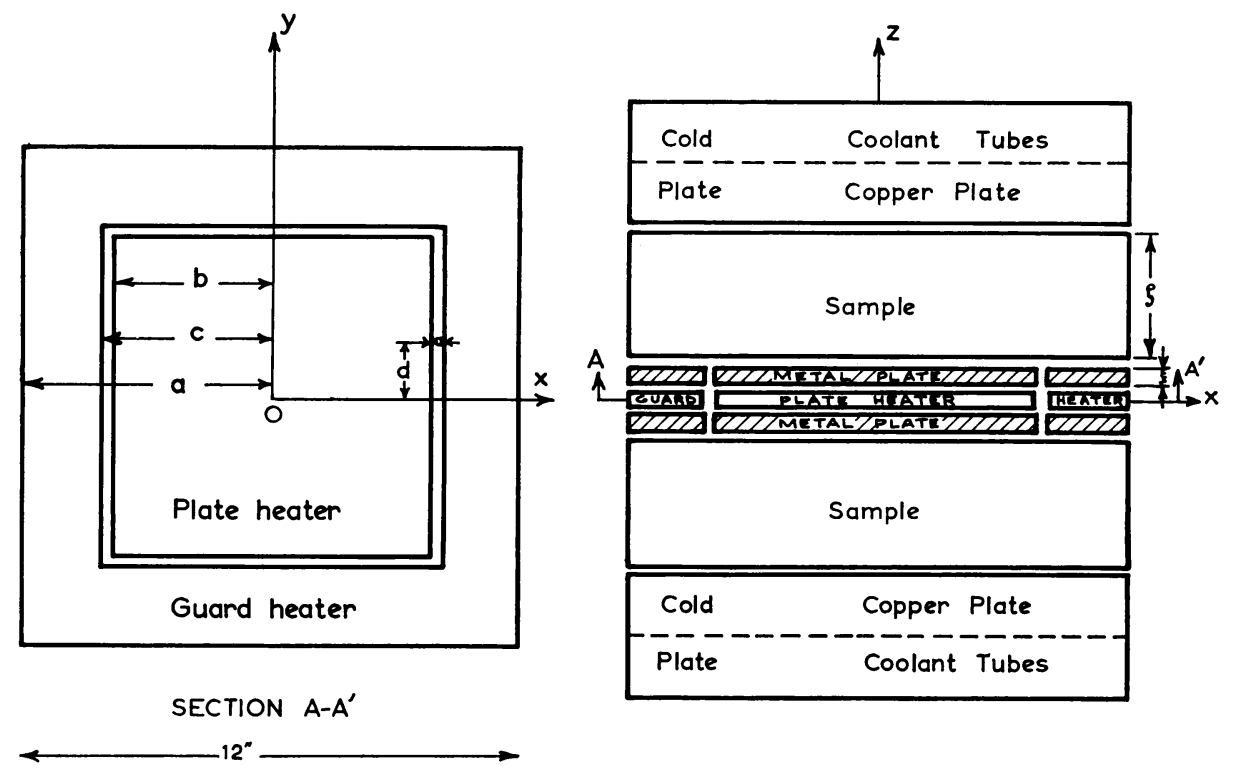

FIG. 1. Schematic diagram of the guarded hot plate apparatus based on the design of the apparatus at Dominion Physical Laboratory. The $z$ scale has been exaggerated $(X 3)$ to show the construction more clearly.

*Received November 23, 1960. 
slabs of the material under test are placed one on either side of an electrically heated metal plate. Outside again are two metal plates kept cold by a flow of water. Thermocouples measure the temperature difference between the hot and cold plates, and the conductivity of the material is calculated from the measured temperature difference, from the known rate of generation of heat per unit area of the hot plate and from the thickness of the specimen.

In this calculation the thermal gradient and the flow of heat are presumed to be everywhere parallel to the axis of the instrument (perpendicular to the plates) but in fact some lateral flow of heat occurs near the outer edges of the hot plate and of the specimen slabs. Consequently the hot plate is provided with a guard ring in imitation of electrical practice. This is a plate in the form of a hollow square closely surrounding the hot plate, fitted with an electric heater and kept at the same temperature as the hot plate. In effect the hot plate becomes merely the central portion of a larger heated plate and the transverse heat flow in its vicinity is much reduced. Of course, an attempt is made to prevent heat passing through the edge faces of the specimens by surrounding the whole apparatus with thermal "insulation", but the thermal conductivity of such material is appreciable. Indeed thermal measurements tend to be much less accurate than the equivalent electrical measurements, mainly because the range of thermal conductivities of available materials is much less than the range of electrical conductivities.

Earlier experimental tests. An experimental check by Robinson and Watson [4] involving 20 different guarded hot plates, all built to the ASTM specifications, found that $75 \%$ of the measured conductivities were in agreement to within $3 \%$ of the mean but some of the results were out by as much as $+13 \%$ and $-16 \%$. Gilbo [5] showed that small temperature differences between hot plate and the guard plate (which will be called "unbalance") could cause considerable errors; for his guarded hot plate $0.2^{\circ} \mathrm{F}$ temperature difference caused $3 \%$ error. He also found that the ambient temperature around the apparatus was another large source of error. Roux et al. [6] investigated errors due to temperature unbalance of the order of $0.03^{\circ} \mathrm{F}$ and reported that the error increased as the conductivity of the sample decreased; for cork (thermal conductivity 0.30 B.t.u. in $/ \mathrm{hr} \mathrm{ft}^{2} \mathrm{~F}$ ) this small unbalance caused an error of more than $0.5 \%$. Pascal [7] in tests on cork $10 \mathrm{~cm}$ thick in his $50 \mathrm{~cm} \times 50 \mathrm{~cm}$ hot plate with gap filled with araldite found that an unbalance equal to $1 \%$ of the temperature difference between hot and cold plates caused an error in conductivity of $35 \%$. Woodside and Wilson [8] investigated unbalance effects and studied the dependence of these errors upon the magnitude and direction of the unbalance, the size and design of the heater plate, the conductivity and thickness of the specimens tested; and the temperature difference between hot and cold plates. Errors as high as $6 \%$ were found under conditions that satisfy the ASTM requirements.

\section{Earlier theoretical assessments.}

\section{Edge heat loss.}

Theoretical assessments that have been made may be divided into two groups; those predicting errors due to edge heat loss (due that is to heat flow through the edge insulation) and those determining the effects of temperature unbalance. Somers and Cyphers [9] did a full analytical treatment of the problem of edge heat losses for a par- 
ticular case, where the temperature of the edge of the sample was equal to that of the cold plate. Dusinberre [10] used a relaxation technique for a similar problem, but allowed for some edge insulation. Pascal [7] also used relaxation to determine the minimum value of the ratio of guard ring width to specimen thickness which would make the transverse flow of heat negligible in the test section of the specimens, but his treatment is two-dimensional not three-dimensional so there is some doubt about the validity of the results.

In the treatment by Woodside [11] the cold plate is taken to be at zero temperature, the hot plate to be at a uniform temperature $\theta_{1}$, and the edge faces of the sample are taken to be at a uniform intermediate temperature $e \theta_{1}$. These assumptions enable a two-dimensional solution to be found by a Schwarz-Christoffel transformation. The solution for the particular case when $e$ is zero agrees well with that of Somers and Cyphers [9], but the assumption of a uniform temperature on the edge face of the specimen seems unrealistic.

\section{Unbalance effects.}

The effect of a temperature difference between the hot plate and its guard ring has been computed by Pascal [7] from the lateral flow of heat through the gap between them. This, however, neglected the lateral flow through the specimens themselves.

Woodside [12] on the other hand based his theory for the error due to unbalance on the simplified system shown in Fig. 2 which takes account of the specimen but ignores the flow of heat across the gap. He justified the extension of the specimens and plate to infinity on each side of the gap and the assumption of two-dimensional heat flow by relaxation calculations (not given in his paper). By applying two successive SchwarzChristoffel transformations, Fig. 2 was transformed to a simpler system for which the solution was readily obtained. As an experimental check the change in the total heat flow due to a temperature difference between hot plate and guard plate was measured on three sets of apparatus and was found to agree within $5 \%$ with the predictions of the foregoing theory.

With the exception of the results of Pascal which are in doubt for the reasons given above, in all the above theoretical approaches it has been assumed that the temperature

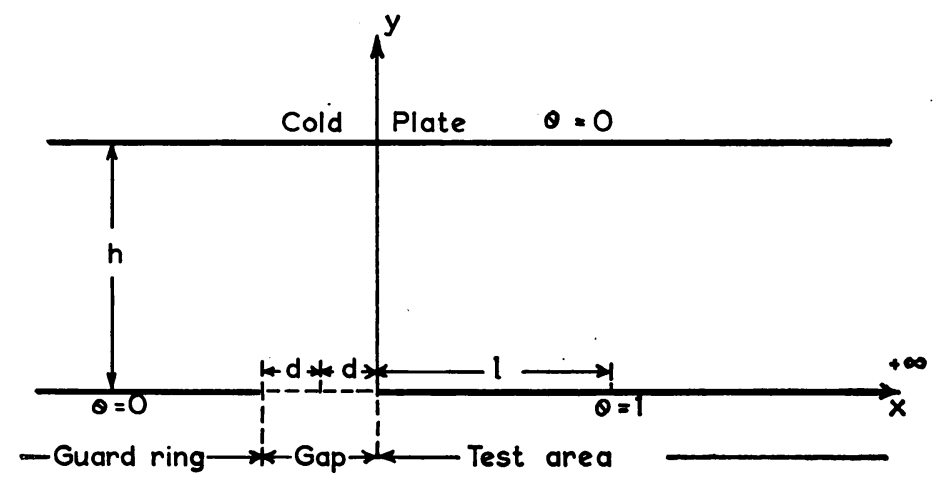

FIG. 2. The artificial plate-specimen temperature system assumed by Woodside [12] to determine unbalance effects. The solution obtained for a uniform temperature over the entire guard plate-hot plate combination $\left(\theta=\theta_{1}\right.$ say) with cold plate maintained at $\theta=0$ is added to this solution. The reduced boundary conditions and dimensions are shown. 
of the hot plate and of the guard plate are uniform over the face of each plate. This assumption is unjustifiable, especially in cases where the guard plate temperature is different from the hot plate temperature or where the temperature maintained outside the edge insulation of the apparatus differs from that of the hot plate, even if some edge insulation is used. To overcome these doubts it was felt that some account should be taken of the heat flow in the metal plate itself. It was found that in so doing it was possible to develop a theory which took into account both edge and unbalance effects and also matched very closely the method by which most guarded hot plates are controlled.

Theoretical analysis. The general arrangement of a guarded hot plate apparatus has already been described in Fig. 1. During operation the cold plates are maintained at some fixed temperature and the heat generated electrically in the hot plate and guard plate is adjusted so that the temperature difference between the central areas of the hot and cold faces of the sample is maintained constant, while the temperature difference between corresponding points on the outside edge of the hot plate and on the inside edge of the guard plate is kept as nearly zero as possible. A theory has been designed to fit this practical model, with but one modification. It has been necessary to assume that the air gap in the metal plates is actually filled with metal, i.e., the plate heater and guard heater are sandwiched between two continuous plates. The effect of this will be discussed later.

Because of the symmetry of the apparatus it is only necessary to consider half of it. The theory therefore discusses the double slab illustrated in Fig. 3, consisting of a metal

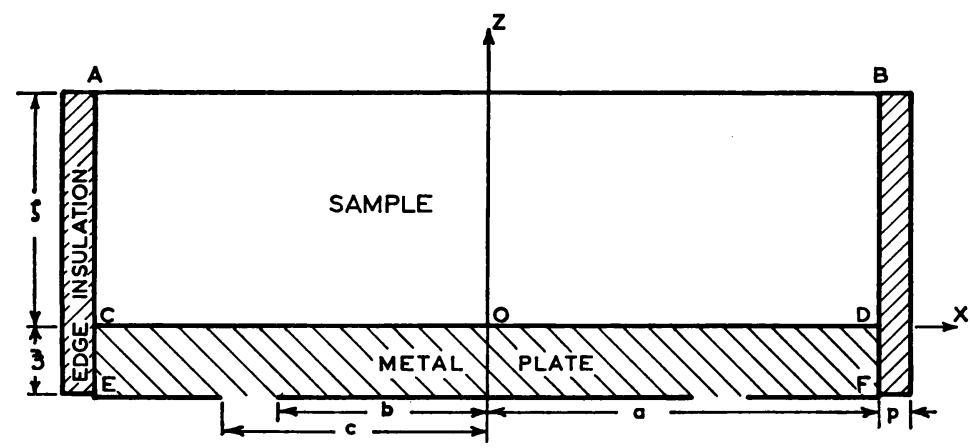

Fig. 3. A cross sectional view of the block used as the basis for the theory. ABDC is the sample and CDFE is the metal plate.

plate of thickness $\xi$ and conductivity $K_{m}$ and the sample of thickness $\zeta$ and conductivity $K_{s}$. Each has a length and width $2 \mathrm{a}$. The origin of coordinate is taken to be the centre point of the interface.

It is assumed that the free face of the specimen is at a constant temperature $T_{0}$ while heat enters the face of the metal plate at a prescribed rate $Q(x, y)$ which depends on position. Around the sides of the compound slab there is assumed to be an insulating layer of conductivity $K_{i}$ and thickness $p$ whose outside face is kept at some temperature taken to be zero for the theory. All other temperatures are measured in degrees Fahren. heit from this value.

In both the sample and the metal plate the temperature obeys Laplace's equation 


$$
\nabla^{2} T=0 .
$$

Boundary conditions have been prescribed for the upper and lower faces. At the interface the temperature and the normal component of heat flow must be continuous. An approximation will be introduced in considering the edge faces. It will be assumed that the flow of heat across these boundaries is proportional to the difference in temperature between the edge and the temperature outside the insulant. This approximation is discussed in a later section. The parameter controlling this will be termed the coefficient of edge heat transfer and, writing $h$ for this, the boundary conditions are

$$
\begin{aligned}
& T=T_{0} \quad z=\zeta, \quad-a<x<a, \quad-a<y<a, \\
& K_{m} \frac{\partial T}{\partial z}=-Q(x, y) \quad z=-\xi, \quad-a<x<a, \quad-a<y<a, \\
& \frac{\partial T}{\partial x}+h T=0 \quad x=+a, \quad-a<y<a, \quad-\xi<z<\zeta, \\
& \frac{\partial T}{\partial x}-h T=0 \quad x=-a, \quad-a<y<a, \quad-\xi<z<\zeta, \\
& \frac{\partial T}{\partial y}+h T=0 \quad y=a, \quad-a<x<a, \quad-\xi<z<\zeta, \\
& \frac{\partial T}{\partial y}-h T=0 \quad y=-a, \quad-a<x<a, \quad-\xi<z<\zeta, \\
& T_{\text {metal }}=T_{\text {sample }} \quad z=0, \quad-a<x<a, \quad-a<y<a, \\
& K_{m}\left(\frac{\partial T}{\partial z}\right)_{\text {metsl }}=K_{s}\left(\frac{\partial T}{\partial z}\right)_{\text {sample }} \quad z=0, \quad-a<x<a, \quad-a<y<a .
\end{aligned}
$$

$Q(x, y)$ is the heat input at the metal face and is defined as follows

$$
\begin{aligned}
& -b<x<b, \quad-b<y<b, \quad Q(x, y)=q_{0} \\
& \left.\begin{array}{c}
-c<y<-b \\
b<y<c
\end{array}\right\}, \quad Q(x, y)=0 \\
& \left.\begin{array}{c}
-a<y<-c \\
c<y<a
\end{array}\right\}, \quad Q(x, y)=q_{1} \\
& \left.\begin{array}{rl}
-c & <x<-b \\
b & <x<c
\end{array}\right\}, \quad-c<y<c, \quad Q(x, y)=0 \\
& \left.\begin{array}{rl}
-a & <y<-c \\
c & <y<a
\end{array}\right\}, \quad Q(x, y)=q_{1} \\
& \left.\begin{array}{rl}
-a & <x<-c \\
c & <x<a
\end{array}\right\}, \quad-a<y<a, \quad Q(x, y)=q_{1} .
\end{aligned}
$$

For either the metal sheet or the sample, the solution of Eq. (1) under the boundary conditions 4 to 7 is of the form (see for instance, Carslaw and Jaeger [13]) 


$$
T=\sum_{r=1}^{\infty} \sum_{s=1}^{\infty}\left(M_{r s} \cosh l_{r s} z+N_{r s} \sinh l_{r s} z\right) \cos \alpha_{r} x \cos \beta_{s} y,
$$

where the coefficients $M$ and $N$ will be different in the sample and in the metal sheet and where

$$
l_{r s}^{2}=\alpha_{r}^{2}+\beta_{s}^{2}
$$

and $\alpha_{r}$ and $\beta_{s}$ are the roots of

$$
\begin{aligned}
& \alpha \tan \alpha a=h, \\
& \beta \tan \beta a=h .
\end{aligned}
$$

It is seen that $\alpha_{r}=\beta_{r}$. Different symbols have been chosen for clarity. In order to determine values of the coefficient (symbolised as $M$ and $N$ ) fitting the boundary conditions noted in Eqs. (2), (3), (8) and (9) it is convenient to note (Carslaw and Jaeger [13]) that when $\alpha_{n}$ is defined in the above manner, the function $f(x)$ obeys the transformation

$$
f(x)=\sum_{n=1}^{\infty} \frac{2\left(\alpha_{n}^{2}+h^{2}\right) \cos \alpha_{n} x}{\left(\alpha_{n}^{2}+h^{2}\right) a+h} \int_{0}^{a} f(x) \cos \alpha_{n} x d x .
$$

The boundary condition (2) states that at the surface $z=\zeta$ the temperature $T$ equals $T_{0}$ so that by applying Eq. (15), in both the $x$ - and $y$-directions, this constant temperature can be written as the function

$$
T_{0}=\sum_{r=1}^{\infty} \sum_{s=1}^{\infty} \frac{4 T_{0}\left(\alpha_{r}^{2}+h^{2}\right)\left(\beta_{s}^{2}+h^{2}\right) \cos \alpha_{r} x \cos \beta_{s} y}{\alpha_{r} \beta_{s}\left\{\left(\alpha_{r}^{2}+h^{2}\right) a+h\right\}\left\{\left(\beta_{s}^{2}+h^{2}\right) a+h\right\}} \sin \alpha_{r} a \sin \beta_{s} a .
$$

Similarly at the surface $z=-\xi$ the heat input is $Q(x, y)$ defined in detail in Eq. (10) and this may in turn be written

$$
\begin{array}{r}
Q(x, y)=\sum_{r=1}^{\infty} \sum_{s=1}^{\infty} \frac{4\left(\alpha_{r}^{2}+h^{2}\right)\left(\beta_{s}^{2}+h^{2}\right) \cos d_{r} x \cos \beta_{s} y}{\alpha_{r} \beta_{s}\left\{\left(\alpha_{r}^{2}+h^{2}\right) a+h\right\}\left\{\left(\beta_{s}^{2}+h^{2}\right) a+h\right\}}\left\{q_{0} \sin \alpha_{r} b \sin \beta_{s} b\right. \\
\left.+q_{1}\left(\sin \alpha_{r} a \sin \beta_{s} a-\sin \alpha_{r} c \sin \beta_{s} c\right)\right\} .
\end{array}
$$

Now at any point within the sample, the temperature distribution can be written [see Eq. (11)] as

$$
T=\sum_{r=1}^{\infty} \sum_{s=1}^{\infty}\left(A_{r s} \cosh l_{r s} z+B_{r s} \sinh l_{r s} z\right) \cos \alpha_{r} x \cos \beta_{s} y
$$

but at the boundary $z=\zeta$ the temperature follows Eq. (16), consequently

$$
A_{r s} \cosh l_{r s} \zeta+B_{r s} \sinh l_{r s} \zeta=\frac{4 T_{0}\left(\alpha_{r}^{2}+h^{2}\right)\left(\beta_{s}^{2}+h^{2}\right) \sin \alpha_{r} a \sin \beta_{s} a}{\alpha_{r} \beta_{s}\left\{\left(\alpha_{r}^{2}+h^{2}\right) a+h\right\}\left\{\left(\beta_{s}^{2}+h^{2}\right) a+h\right\}} .
$$

Again, at any point within the metal plate

$$
T=\sum_{r=1}^{\infty} \sum_{s=1}^{\infty}\left(C_{r s} \cosh l_{r s} z+D_{r s} \sinh l_{r s} z\right) \cos \alpha_{r} x \cos \beta_{s} y .
$$

The value of $Q$ at the boundary $z=-\xi$ can be deduced from this, using (3) and comparison with expansion (17) shows that 
$-C_{r s} \sinh l_{r s} \xi+D_{r s} \cosh l_{r s} \xi$

$=-\frac{4\left(\alpha_{r}^{2}+h^{2}\right)\left(\beta_{s}^{2}+h^{2}\right)\left\{q_{0} \sin \alpha_{r} b \sin \beta_{s} b+q_{1}\left(\sin \alpha_{r} a \sin \beta_{s} a-\sin \alpha_{r} c \sin \beta_{s} c\right)\right\}}{K_{m} l_{r s} \alpha_{r} \beta_{s}\left\{\left(\alpha_{r}^{2}+h^{2}\right) a+h\right\}\left\{\left(\beta_{s}^{2}+h^{2}\right) a+h\right\}}$.

Further, the boundary conditions (8) and (9) applied to Eqs. (18) and (20) give

$$
\begin{aligned}
A_{r s} & =C_{r s}, \\
K_{m} D_{r s} & =K_{s} B_{r s} .
\end{aligned}
$$

Relations (19), (21), and (22) allow the coefficients $A, B, C$ and $D$ to be expressed in terms of the known constants, so that within the metal sheet

$T=\sum_{r=1}^{\infty} \sum_{s=1}^{\infty}$

$\frac{4\left(\alpha_{r}^{2}+h^{2}\right)\left(\beta_{s}^{2}+h^{2}\right) \cos \alpha_{r} x \cos \beta_{s} y}{\alpha_{r} \beta_{s}\left\{\left(\alpha_{r}^{2}+h^{2}\right) a+h\right\}\left\{\left(\beta_{s}^{2}+h^{2}\right) a+h\right\}\left(K_{m} \sinh l_{r s} \xi \sinh l_{r s} \zeta+K_{s} \cosh l_{r s} \xi \cosh l_{r s} \zeta\right)}$

$\cdot\left\{\left[\frac{1}{l_{r s}}\left\{q_{0} \sin \alpha_{r} b \sin \beta_{s} b+q_{1}\left(\sin \alpha_{r} a \sin \beta_{s} a-\sin \alpha_{r} c \sin \beta_{s} c\right)\right\} \sinh l_{r s} \zeta\right.\right.$

$\left.+K_{s} T_{0} \sin \alpha_{r} a \sin \beta_{s} a \cosh l_{r s} \xi\right] \cosh l_{r s} z$

$+\left[-\frac{K_{s}}{K_{m} l_{r s}}\left\{q_{0} \sin \alpha_{r} b \sin \beta_{s} b+q_{1}\left(\sin \alpha_{r} a \sin \beta_{s} a-\sin \alpha_{r} c \sin \beta_{s} c\right)\right\} \cosh l_{r s} \zeta\right.$

$\left.\left.+K_{s} T_{0} \sin \alpha_{r} a \sin \beta_{s} a \sinh l_{r s} \xi\right] \sinh l_{r s} z\right\}$

and within the sample

$$
\begin{aligned}
T & =\sum_{r=1}^{\infty} \sum_{s=1}^{\infty} \\
& : \frac{4\left(\alpha_{r}^{2}+h^{2}\right)\left(\beta_{s}^{2}+h^{2}\right) \cos \alpha_{r} x \cos \beta_{s} y}{\left.\left(\alpha_{r}^{2}+h^{2}\right) a+h\right\}\left\{\left(\beta_{s}^{2}+h^{2}\right) a+h\right\}\left(K_{m} \sinh l_{r s} \xi \sinh l_{r s} \zeta+K_{s} \cosh l_{r s} \xi \cosh l_{r s} \zeta\right)} \\
& \cdot\left\{\left[\frac{1}{l_{r s}}\left\{q_{0} \sin \alpha_{r} b \sin \beta_{s} b+q_{1}\left(\sin \alpha_{r} a \sin \beta_{s} a-\sin \alpha_{r} c \sin \beta_{s} c\right)\right\} \sinh l_{r s} \zeta\right.\right. \\
+ & \left.K_{s} T_{0} \sin \alpha_{r} a \sin \beta_{s} a \cosh l_{r s} \xi\right] \cosh l_{r s} z \\
+ & {\left[-\frac{1}{l_{r s}}\left\{q_{0} \sin \alpha_{r} b \sin \beta_{s} b+q_{1}\left(\sin \alpha_{r} a \sin \beta_{s} a-\sin \alpha_{r} c \sin \beta_{s} c\right)\right\} \cosh l_{r s} \zeta\right.} \\
+ & \left.\left.K_{m} T_{0} \sin \alpha_{r} a \sin \beta_{s} a \sinh l_{r s} \xi\right] \sinh l_{r s} z\right\} .
\end{aligned}
$$

Thus for any point $(x, y, z)$ in the sample or in the metal plate, the temperature is given by

$$
T=\mu(x, y, z) q_{0}+\nu(x, y, z) q_{1}+\lambda(x, y, z) T_{0},
$$

where $\mu(x, y, z), \nu(x, y, z)$ and $\lambda(x, y, z)$ are, for any particular guarded hot plate functions of position only. In operating the apparatus, as pointed out earlier, the heat inputs into the hot plate and the guard plates are controlled to maintain a fixed temperature difference between the central areas of the hot and cold faces of the sample. For the 
theory, as it is here, central points only are used. The theory may however be modified for any point or set of points. At the same time the temperature difference between two points, one on the edge of the guard plate and one on the edge of the hot plate, is maintained zero. Applying these conditions to Eq. (25), two equations

$$
T_{0}+\Delta T=\mu(0,0,0) q_{0}+\nu(0,0,0) q_{1}+\lambda(0,0,0) T_{0}
$$

and

$$
\begin{aligned}
\{\mu(c, d, 0)-\mu(b, d, 0)\} q_{0}+\{\nu(c, d, 0)-\nu(b, d, 0)\} q_{1} \\
+\{\lambda(c, d, 0)-\lambda(b, d, 0)\}=0
\end{aligned}
$$

are obtained and hence it is possible to determine $q_{0}$ and $q_{1}$ for any $T_{0}$. Variation of $T_{0}$ is effectively the same as varying the temperature of the controlled atmosphere surrounding the apparatus. The measured thermal conductivity of the sample is then obtained from the heat input/sq. foot of the heater, the dimensions of the apparatus, the thickness of the sample and the temperature difference across the sample

$$
\left(K_{s}\right)_{\text {meas. }}=\frac{q_{0} b^{2} \zeta}{\frac{1}{4}(b+c)^{2} \Delta T} .
$$

Unbalance effects. The theory as so far described covers all variations provided the apparatus is in balance, i.e., no temperature difference between a pair of points on the outside edge of the hot plate and the inside of the guard plate. In practice a gap fitted with a poor conductor (in most cases-air) separates the guard plate from the hot plate, but in the theory above no such gap exists.

To match the theory more closely with the apparatus as it is in practice, it is necessary when determining unbalance effects to consider the problem in two parts: (a) the effect of the correct heat flux in the metal plate and (b) the correction for the effect of the temperature difference across the gap on the sample itself.

(a) Thus, if $T^{\prime}$ is the temperature difference across the actual gap (at the pair of points) and the gap is filled with material of conductivity $K^{\prime}$, then the heat flows would be the same as if the temperature difference between the same two points in the solid metal plate were

$$
T^{\prime \prime}=T^{\prime} \frac{K^{\prime}}{K_{m}}
$$

For the determination of the measured thermal conductivity in this case $T^{\prime \prime}$ replaces the zero in the right hand side of Eq. (27) and this is combined with Eq. (26) to determine a $q_{0}$. This $q_{0}$ thus takes lateral heat flux in the metal plate into account but must be corrected for effects in the sample.

(b) In the sample itself the actual temperature difference $T^{\prime}$ applies. Consider the sample alone with the boundary conditions (4)-(7) as before, but with

$$
\begin{array}{llll}
T=0 & z=\zeta & -a<x<a & -a<y<a, \\
T=T(x, y) & z=0 & -a<x<a & -a<y<a,
\end{array}
$$

where $T(x, y)$ is zero if $|x|$ and $|y|$ are both less than $\frac{1}{2}(b+c)$ otherwise it is equal to $T^{\prime}$. ( $T^{\prime}$ is positive when guard plate is the hotter.) 
The solution obtained is

$$
\begin{aligned}
T_{a}=\sum_{m=1}^{\infty} \sum_{n=1}^{\infty} \frac{4 T^{\prime}\left(\alpha_{n}^{2}+h^{2}\right)\left(\beta_{m}^{2}+h^{2}\right) \cos \alpha_{n} x \cos \beta_{m} y \sinh l_{m n}(\zeta-z)}{\alpha_{n} \beta_{m}\left\{\left(\alpha_{n}^{2}+h^{2}\right) a+h\right\}\left\{\left(\beta_{m}^{2}+h^{2}\right) a+h\right\} \sinh l_{m n} \zeta} \\
\times\left\{\sin \alpha_{n} a \sin \beta_{m} a-\sin \frac{1}{2} \alpha_{n}(b+c) \sin \frac{1}{2} \beta_{m}(b+c)\right\} .
\end{aligned}
$$

The total heat entering the sample from the hot plate must therefore be

$$
q_{0} b^{2}+K_{s} \int_{-(b+c) / 2}^{(b+c) / 2} \int_{-(b+c) / 2}^{(b+c) / 2}\left(\frac{\partial T_{a}}{\partial z}\right)_{z=0} d x d y
$$

and the measured conductivity will be

$$
\begin{aligned}
& \left(K_{s}\right)_{\mathrm{meas}}=\frac{4 \zeta}{(b+c)^{2} \Delta T}\left\{b^{2} q_{0}\right. \\
& \quad-\sum_{m=1}^{\infty} \sum_{n=1}^{\infty} \frac{16 K_{s} T^{\prime} l_{m n}\left(\alpha_{n}^{2}+h^{2}\right)\left(\beta_{m}^{2}+h^{2}\right) \sin \frac{1}{2} \alpha_{n}(b+c) \sin \frac{1}{2} \beta_{m}(b+c)}{\alpha_{n}^{2} \beta_{m}^{2}\left\{\left(\alpha_{n}^{2}+h^{2}\right) a+h\right\}\left\{\left(\beta_{m}^{2}+h^{2}\right) a+h\right\}} \operatorname{coth} l_{m n} \zeta \\
& \quad \times\left\{\sin \alpha_{n} a \sin \beta_{m} a-\sin \frac{1}{2} \alpha_{n}(b+c) \sin \frac{1}{2} \beta_{m}(b+c)\right\} .
\end{aligned}
$$

The coefficient of edge heat transfer. In a system in which the insulation is in contact with only one other material a simplification often used is to assume that the insulation may be replaced by an extremely thin layer of material of almost zero conductivity, i.e., the flow of heat through the insulation is taken to be perpendicular to the interface between insulation and the material being insulated. In this case the boundary condition used pertains to this interface and is taken in the form

$$
\frac{d T}{d n}=h\left(T-T_{w}\right),
$$

where $n$ is in the direction normal to this interface and $T_{10}$ is the temperature at which the outside of the insulation is maintained. A reasonable approximation for the coefficient of edge heat transfer is then

$$
h=\frac{K_{i}}{K d}
$$

provided that there is good contact between the two materials, and that the temperature gradient through the insulation is much greater perpendicular to the interface than that parallel to the interface. In Eq. (36) $K_{i}$ is the thermal conductivity of the insulation, $K$ is the conductivity of the other material and $d$ is the thickness of the insulating layer.

In the guarded hot plate the insulating layer is, however, in contact with both the sample in the apparatus and with the metal of the guard plate. As well as this the temperature gradient parallel to the interface will be comparable with that through the insulation, thus any attempt at defining $h$ as in Eq. (36) could lead to considerable inaccuracy. Nevertheless it is necessary to obtain a uniform value of $h$ over the entire edge matching as closely as possible the edge heat losses for any particular set up in order that the equations may be solved. This value of $h$ was determined as follows. The arrangement was simplified by working in two dimensions only. The sample/hot plate block $L M M^{\prime} L^{\prime}$ (Fig. 4) and the insulation $M M^{\prime} V V^{\prime}$ were considered separately. It was assumed that 


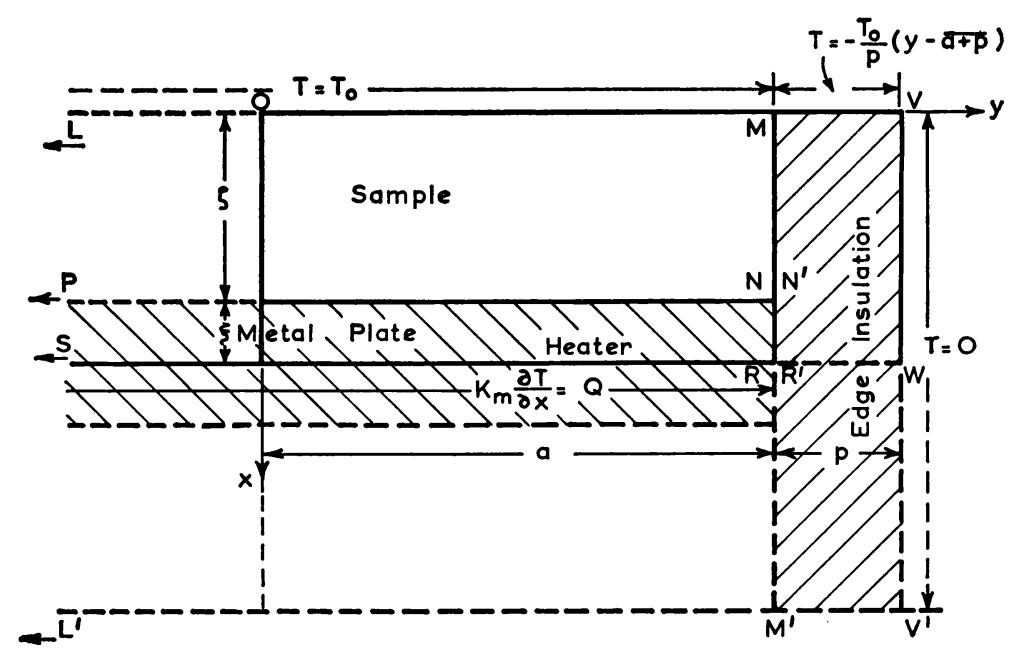

FIG. 4. The boundary conditions assumed for the determination of the coefficient of edge heat transfer, $h$.

the block $L M M^{\prime} L^{\prime}$ was edged by a thin layer with coefficient of edge heat transfer $h$ in place of the layer of insulation and using this the heat flow over any section of the face $M M^{\prime}$ was determined. The temperature at the point $N$ was also determined. The block of insulation was then considered and it was assumed that the heat flowing over any section of the face $M M^{\prime}$ of this block matched that flowing out over the same section of the face $M M^{\prime}$ of block $L M M^{\prime} L^{\prime}$. The temperature at the point $N^{\prime}$ was then determined and this temperature was equated to the temperature determined for the point $N$ of the block $L M M^{\prime} L^{\prime}$.

For the block LMRS (Fig. 4) the boundary conditions are (assuming that the coefficient of edge heat transfer is $h$ )

$$
\begin{aligned}
& \text { at } x=0 \\
& T=T_{0}, \\
& \text { at } x=\zeta \quad K_{m}\left(\frac{\partial T}{\partial x}\right)_{m}=K_{s}\left(\frac{\partial T}{\partial x}\right)_{s} \text {, } \\
& \text { at } x=\zeta \\
& T_{m}=T_{s}, \\
& \text { at } x=\zeta+\xi \quad K_{m} \frac{\partial T}{\partial x}=Q, \\
& \text { at } y=-a \quad \frac{\partial T}{\partial y}-h T=0, \\
& \text { at } y=a \quad \frac{\partial T}{\partial y}+h T=0,
\end{aligned}
$$

where $K_{m}$ is the conductivity of the metal plate, $K_{s}$ is the conductivity of the sample and the subscripts $m$ and $s$ refer to the metal and the sample respectively. The heat input $Q$ is so chosen that if the edge insulation were perfect the temperature difference across the sample would be $40^{\circ} \mathrm{F}$. Similar boundary conditions apply for the block $L^{\prime} M^{\prime} R S$.

Thus solving Laplace's equation in the block $L M M^{\prime} L^{\prime}$ with these boundary con- 
ditions the temperature at any point within the block is given by

$$
\begin{aligned}
T & =\sum_{r=1}^{\infty}\left\{A_{r} \cosh \alpha_{r}(x-\zeta)+\frac{K_{m}}{K_{s}} B_{r} \sinh \alpha_{r}(x-\zeta)\right\} \cos \alpha_{r} y \quad 0 \leq x \leq \zeta \\
& =\sum_{r=1}^{\infty}\left\{A_{r} \cosh \alpha_{r}(x-\zeta)+B_{r} \sinh \alpha_{r}(x-\zeta)\right\} \cos \alpha_{r} y \quad \zeta \leq x \leq \zeta+\xi \\
& =\sum_{r=1}^{\infty}\left\{A_{r} \cosh \alpha_{r}(x-\overline{\zeta+2 \xi})-B_{r} \sinh \alpha_{r}(x-\overline{\zeta+2 \xi})\right\} \cos \alpha_{r} y \\
& =\sum_{r=1}^{\infty}\left\{A_{r} \cosh \alpha_{r}(x-\overline{\zeta+2 \xi})-\frac{K_{m}}{K_{s}} B_{r} \sinh \alpha_{r}(x-\overline{\zeta+2 \xi})\right\} \cos \alpha_{r} y \\
\zeta+2 \xi \leq x \leq 2(\zeta+\xi), &
\end{aligned}
$$

where

$$
\begin{aligned}
& A_{r}=\frac{2 h\left(K_{s} T_{0} \cosh \alpha_{r} \xi+\frac{Q}{\alpha_{r}} \sinh \alpha_{r} \zeta\right)}{\left\{\left(\alpha_{r}^{2}+h^{2}\right) a+h\right\} \cos \alpha_{r} a\left(K_{s} \cosh \alpha_{r} \zeta \cosh \alpha_{r} \xi+K_{m} \sinh \alpha_{r} \xi \sinh \alpha_{r} \xi\right)} \\
& B_{r}=\frac{2 h\left(-K_{s} T_{0} \sinh \alpha_{r} \xi+\frac{K_{s} Q}{K_{m} \alpha_{r}} \cosh \alpha_{r} \xi\right)}{\left\{\left(\alpha_{r}^{2}+h^{2}\right) a+h\right\} \cos \alpha_{r} a\left(K_{s} \cosh \alpha_{r} \zeta \cosh \alpha_{r} \xi+K_{m} \sinh \alpha_{r} \zeta \sinh \alpha_{r} \xi\right)}
\end{aligned}
$$

and by differentiating this with respect to $y$ and multiplying by the applicable value of the conductivity the heat flow over any section of the boundary $M M^{\prime}$ may be obtained

$$
\begin{array}{r}
K(x)\left(\frac{\partial T}{\partial y}\right)_{y=a}=\sum_{r=1}^{\infty} K_{s} \alpha_{r} \sin \alpha_{r} a\left\{A_{r} \cosh \alpha_{r}(x-\zeta)+\frac{K_{m}}{K_{s}} B_{r} \sin \alpha_{r}(x-\zeta)\right\} \\
=\sum_{r=1}^{\infty} K_{m} \alpha_{r} \sin \alpha_{r} a\left\{A_{r} \cosh \alpha_{r}(x-\zeta)+B_{r} \sinh \alpha_{r}(x-\zeta)\right\} \\
\quad \zeta \leq \leq \zeta \\
=\sum_{r=1}^{\infty} K_{m} \alpha_{r} \sin \alpha_{r} a\left\{A_{r} \cosh \alpha_{r}(x-\overline{\zeta+2 \xi})-B_{r} \sinh \alpha_{r}(x-\overline{\zeta+2 \xi})\right\} \\
=\sum_{r=1}^{\infty} K_{s} \alpha_{r} \sin \alpha_{r} a\left\{A_{r} \cosh \alpha_{r}(x-\overline{\zeta+2 \xi})-\frac{K_{m}}{K_{s}} B_{r} \sinh \alpha_{r}(x-\zeta+2 \xi)\right\} \\
\zeta+2 \xi \leq x \leq 2(\zeta+\xi)
\end{array}
$$

while the temperature at $N(y=a, x=\zeta)$ is

$$
T_{N}=\sum_{r=1}^{\infty} A_{r} \cos \alpha_{r} a .
$$

For the edge insulation $M V V^{\prime} M^{\prime}$ it is assumed that

$$
\left.\begin{array}{ll}
\text { at } \quad y=a+p \\
\text { at } \quad x=0 \\
x=2(\zeta+\xi)
\end{array}\right\} \quad T=\frac{T}{p}(-y+a+p)
$$


while at

$$
y=a \quad K_{i}\left(\frac{\partial T}{\partial y}\right)_{\substack{i \\ y=a}}=K(x)\left(\frac{\partial T}{\partial y}\right)_{y=a},
$$

where $K(x)(\partial T / \partial y)_{y=a}$ is defined above in Eq. (46) and the subscript $i$ pertains to the insulation.

The temperature assumed along the edges $M V$ and $M^{\prime} V^{\prime}$ of this block, as given in (49) is from necessity an approximation and will give for any simple arrangement of insulation a slightly low value of $h$. If the entire hot plate assembly is covered by the same thickness of insulation the temperature variation across these faces does not differ greatly from the linear variation assumed. A relaxation technique was used to determine this temperature variation in several cases.

Solving Laplace's equation in the block $M V V^{\prime} M^{\prime}$ under the boundary conditions (48), (49) and (50) the temperature at any point within the block is found

$$
\begin{aligned}
T=\frac{T_{0}}{p}( & -y+a+p) \\
& -\sum_{n=1}^{\infty} \sum_{r=1}^{\infty}\left\{\frac{4(\zeta+\xi) \alpha_{r} \sin \alpha_{r} a}{n \pi K_{i}\left[n^{2} \pi^{2}+4 \alpha_{r}^{2}(\zeta+\xi)^{2}\right]}\right. \\
& \cdot\left[4 K_{m} \alpha_{r}(\zeta+\xi) \sin \frac{n \pi}{2}\left(A_{r} \sinh \alpha_{r} \xi+B_{r} \cosh \alpha_{r} \xi\right)+n \pi(1-\cos n \pi)\right. \\
& \left.\cdot\left(-K_{s} A_{r} \cosh \alpha_{r} \zeta+K_{m} B_{r} \sinh \alpha_{r} \zeta-\left(K_{m}-K_{s}\right) A_{r} \cos \frac{n \pi \zeta}{2(\zeta+\xi)}\right)\right] \\
+ & \left.\frac{4(\zeta+\xi) T_{0}}{n^{2} \pi^{2} p}\right\} \frac{\sinh \left\{\frac{n \pi(a+p-y)}{2(\zeta+\xi)}\right\} \sin \frac{n \pi x}{2(\zeta+\xi)}}{\cosh \frac{n \pi p}{2(\zeta+\xi)}}
\end{aligned}
$$

and thus the temperature at the point $N^{\prime}(y=a, x=\zeta)$ is

$$
\begin{aligned}
T=T_{0} & -\sum_{n=1}^{\infty} \frac{4(\zeta+\xi) T_{0}}{n^{2} \pi^{2} p} \tanh \frac{n \pi p}{2(\zeta+\xi)} \sin \frac{n \pi \zeta}{2(\zeta+\xi)} \\
- & \frac{1}{K_{i}} \sum_{n=1}^{\infty} \sum_{r=1}^{\infty}\left\{\frac{4(\zeta+\xi) \alpha_{r} \sin \alpha_{r} a}{n \pi\left[n^{2} \pi^{2}+4 \alpha_{r}^{2}(\zeta+\xi)^{2}\right]}\right. \\
& \cdot\left[4 K_{m} \alpha_{r}(\zeta+\xi) \sin \frac{n \pi}{2}\left(A_{r} \sinh \alpha_{r} \xi+B_{r} \cos \alpha_{r} \xi\right)+n \pi(1-\cos n \pi)\right. \\
& \left.\left.\cdot\left(-K_{s} A_{r} \cosh \alpha_{r} \zeta+K_{m} B_{r} \sinh \alpha_{r} \zeta-\left(K_{m}-K_{s}\right) A_{r} \cos \frac{n \pi \zeta}{2(\zeta+\xi)}\right)\right]\right\} \\
\times & \tanh \frac{n \pi p}{2(\zeta+\xi)} \sin \frac{n \pi \zeta}{2(\zeta+\xi)} .
\end{aligned}
$$

As $N$ and $N^{\prime}$ are in fact the same point the temperatures as defined by (47) and (52) should be the same and thus by equating these $h$ may be computed in terms of the other constants. This equation is however not linear in $h$, whereas it is linear in $K_{i}$ hence it is much more convenient to choose an $h$ and then solve for $K_{i}$. The variation of $K_{i}$ 
with $h$ was determined for various discrete values of the dimensions and for various materials as heater plate and sample. In all cases it was assumed that the apparatus was 1 foot square, i.e., $a=0.5$ foot.

The results of these computations show that provided the thickness of insulation is greater than the thickness of one sample $(p>\zeta), h$ does not vary with insulation thickness, neither does it vary significantly with the conductivity of the sample itself for the range of values considered $\left(h<.04 \mathrm{ft}^{-1}, .02 \leq K_{\mathrm{s}} \leq .10 \mathrm{Btu} / \mathrm{ft}{ }^{\circ} \mathrm{Fhr}, .01 \leq K_{i} \leq\right.$ $\left..04 \mathrm{Btu} / \mathrm{ft}^{\circ} \mathrm{Fhr} K_{m}=0\left(10^{3}\right) K_{\mathrm{s}}\right)$. It was found that $h$ is approximately proportional to the conductivity of the insulation $K_{i}$, inversely proportional to the conductivity of the metal plate $K_{m}$, and for the $Q$ used here it is inversely proportional to $T_{0}+40$ for the range $-35 \leq T_{0} \leq 0^{\circ} \mathrm{F}$.

The variation of $h$ with $\xi$ and $\zeta$ is rather more complicated but is given by

$$
h \propto \phi(\zeta, \xi),
$$

where $\phi(\zeta, \xi)$ is given for various discrete values of $\zeta, 1 / 16^{\prime \prime} \leq \xi \leq 3 / 8^{\prime \prime}$ in Fig. 5. This may all be combined in the single formula

$$
h=\frac{K_{i} \phi(\zeta, \xi)}{K_{m}\left(T_{0}+40\right)}
$$

which defines $h$ to within $7 \%$ of the computed values.

To justify to some degree the method of determining the value of $h, K_{i}$ has been

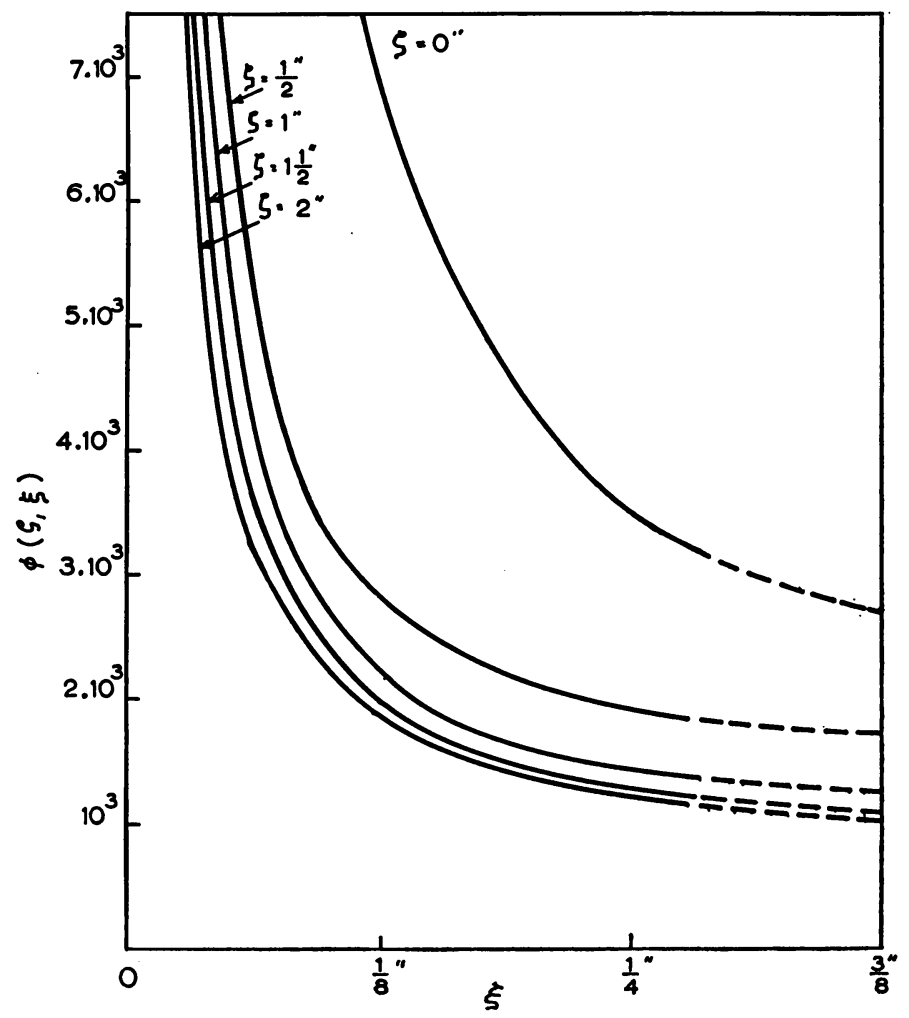

FIG. 5. The variation of $\phi(\zeta, \xi)$.with $\xi$ for discrete values of $\zeta$. 
determined by equating the temperatures at several other points at the interface between the two blocks instead of at $N$ and $N^{\prime}$. The values obtained have been compared with those obtained above and it was found that for all the values of $K_{i}$ likely to be met in practice, i.e., $0.01 \leq K_{i} \leq 0.04, \mathrm{Btu} / \mathrm{ft}{ }^{\circ} \mathrm{F} / \mathrm{hr}$. the variation of $h$ across the edge of the sample or of the metal plate for a particular $K_{i}$ is less than $6 \%$ of the value determined above. As in the simplified heating system used in this section (no guard heater) a variation of $h$ by $10 \%$ merely alters the temperature difference across the centre of the sample from hot to cold plate by less than $0.3 \%$, it is to be expected that in the calculations for the full apparatus a variation of $h$ of the order of $10 \%$ will have a negligible effect and hence there is little justification for any attempt to determine $h$ to a higher degree of accuracy.

Conclusions. The theory developed above thus effectively matches most aspects of the guarded hot plate and its operation. It is possible to allow for the variation of any of the following

(a) The outer dimensions of the guard plate.

(b) The inner dimensions of the guard plate heater.

(c) The outer dimensions of the hot plate heater.

(d) The thickness of the metal plate.

(e) The material of the metal plate.

(f) The thickness of the sample.

(g) The material of the sample.

(h) The insulation around the apparatus.

(i) The temperature difference between hot and cold plates.

(j) The temperature of the cold plate (this effectively alters the temperature of the surroundings).

(k) The positions of the thermocouples controlling balance.

(l) The amount of unbalance.

(m) The gap width and the material with which the gap is filled.

This theory was not intended to be used in the direct measurement of thermal conductivity, but rather as a full check of the accuracy of the application of the simple linear flow formula under all conditions. The results will be used in the designing of an apparatus in which the total error will be minimised.

The double series (24) is rather slowly coverging and preliminary computations showed that although 100 terms were sufficient to give the temperature to 4 decimal places, insufficient accuracy was obtained to permit $q_{0}$ and $q_{1}$ to be computed with any certainty.

It is intended to obtain a full solution for a 1 foot square guarded hot plate on a digital computer, but at the present time access to one is being awaited. Once a full solution has been obtained for an apparatus of one size, the solution will be equally applicable to guarded hot plates of other sizes by scaling the results.

Acknowledgements. The author would like to thank Miss L. Davey and Mrs. J. Barnard for assistance with the computations, and Mr. J. E. Mautner, who designed and now operates the guarded hot plate apparatus at Dominion Physical Laboratory, for much of the practical details. He would also like to express his thanks to those members of the staff of Dominion Physical Laboratory and Applied Mathematics Laboratory who took part in useful discussions during the progress of this project. 


\section{REFERENCES}

1. Method of test for thermal conductivity of materials by means of the guarded hot plate (C 177-45), 1955 Book of ASTM Standards, Part 3, p. 1084

2. Method of test for thermal conductivity of building materials by means of the guarded hot plate, Laboratorio Nacional de Engenharia Civil, Ministerio das Obras Publicas, Lisbon, Portugal, May 1954

3. Definitions of heat insulating terms and methods of determining thermal conductivity and solar reflectivity, Standard No. 874, British Standards Institution, 1956

4. H. E. Robinson and T. W. Watson, Interlaboratory comparison of thermal conductivity determinations with guarded hot plates, Symposium on Thermal Insulating Materials, Am. Soc. Testing Materials, Special Technical Publication, No. 119, p. 36 (1951)

5. C. F. Gilbo, Experiments with a guarded hot plate thermal conductivity set, Symposium on Thermal Insulating Materials, Am. Soc. Testing Materials, Special Technical Publication No. 119, p. 45 (1951)

6. A. J. A. Roux, S. J. Richards, and W. M. H. Rennhackkamp, The Measurement of the thermal properties of building materials-the thermal conductivity of thin 12" square samples, Series DR-6, South African Council for Scientific and Industrial Research, National Building Research Inst., Nov. 1950

7. A. Pascal, La mesure de la conduction thermique des materiaux du batiment, Inst. Technique du Batiment et des Travaux Publics, No. 90, pp. 583-598 (June 1955)

8. W. Woodside and A. G. Wilson, Unbalance errors in guarded hot plate measurements, Symposium on Thermal Conductivity Measurements and Applications of Thermal Insulations, Am. Soc. Testing Materials, Special Technical Publication, No. 217, p. 32 (1957)

9. E. V. Somers and J. A. Cyphers, Analysis of errors in measuring thermal conductivities of thermal insulating materials, Rev. Sci. Instr. 22, No. 8, 583-585 (1951)

10. G. M. Dusinberre, Further analysis of errors of the guarded hot plate, Rev. Sci. Instr. 23, No. 11, 649 (1952)

11. W. Woodside, Analysis of errors due to edge heat loss in guarded hot plates, Symposium on Thermal Conductivity Measurements and Applications of Thermal Insulations, Am. Soc. Testing Materials, Special Technical Publication, No. 217, p. 49 (1957)

12. W. Woodside, Deviations from one-dimensional flow in guarded hot plate measurements, Rev. Sci. Instr. 28, No. 12, 1033-1037 (1957)

13. H. S. Carslaw and J. C. Jaeger, Conduction of heat in solids, Clarendon Press, Oxford, 1st ed., 1948, p. 99 